\title{
Endovascular repair of the thoracic aorta in the post-FDA approval era
}

\author{
Joshua D. Adams, MD, ${ }^{\mathrm{a}, \mathrm{b}}$ John F. Angle, MD, ${ }^{\mathrm{a}}$ Alan H. Matsumoto, MD, ${ }^{\mathrm{a}}$ Benjamin B. Peeler, MD, ${ }^{\mathrm{b}}$ Bulent Arslan, MD, \\ Kenneth J. Cherry, MD, ${ }^{b}$ John A. Kern, MD, ${ }^{b}$ and Michael D. Dake, MD ${ }^{a}$
}

\begin{abstract}
Objective: Endovascular repair of thoracic aortic disease is rapidly progressing as an alternative to open surgical therapy. In March of 2005, the Gore TAG thoracic endoprosthesis (W. L. Gore \& Associates, Inc, Flagstaff, Ariz) received Food and Drug Administration (FDA) approval for the treatment of descending thoracic aortic aneurysms. Subsequently, off-label use of the technology expanded to include additional thoracic aortic diseases. The purpose of this study was to examine whether the outcomes with this device changed after the inclusion and exclusion criteria of FDA-controlled trials no longer governed patient selection.
\end{abstract}

Methods: A retrospective analysis was performed on all patients who underwent endovascular repair of the thoracic aorta with the Gore TAG device at our institution between March 23, 2005, and September 8, 2006.

Results: Fifty consecutive patients with a broad range of aortic pathologic conditions were included in the study. The results in this group compared with those of the phase II trial included the following: length of stay, 7.5 versus 7.6 days $(P=.97)$; intensive care unit stay, 3.7 versus 2.6 days $(P=.61)$; 30 -day mortality, $2.0 \%$ versus $1.5 \%$ $(P=.68)$; spinal cord injury, $2 \%$ versus $3 \%(P=.89)$; stroke, $4 \%$ versus $4 \%(P=.67)$; early endoleaks, $26 \%$ versus $4 \%(P<.01)$; and late endoleaks, $18 \%$ versus $7 \%(P=.08)$. At 1 year, overall survival was $92 \%$ compared with $82 \%$ in the phase II trial.

Conclusions: In the post-FDA approval era, endovascular stent-graft therapy is frequently applied to patients with more challenging thoracic aortic anatomy and a wide range of pathologic conditions. Our results in this group are similar to outcomes reported for patients with descending thoracic aortic aneurysm exclusively.

In the early 1990 s, treatment of thoracic aortic aneurysms entered the endovascular era. ${ }^{1}$ Over the next decade, results steadily improved as strict inclusion and exclusion criteria were used to guide patient selection. ${ }^{2-5}$ Currently, one device, the Gore TAG thoracic endoprosthesis (W. L. Gore \& Associates, Flagstaff, Ariz) is approved by the Food and Drug Administration (FDA) and commercially available in the United States for the treatment of descending thoracic aortic aneurysms. At least three other devices are in clinical trials. With commercial availability, endovascular repair of thoracic aortic disease has markedly increased. Off-label use has rapidly expanded to include additional thoracic aortic abnormalities such as pseudoaneurysm, type B aortic dissection, traumatic aortic disruption, complicated penetrating ulcer, intramural hematoma, and lesions related to connective tissue disorders. ${ }^{6,7}$

The purpose of this study was to evaluate whether "postFDA approval era" outcomes are affected by the removal of

From the Departments of Radiology ${ }^{\mathrm{a}}$ and Surgery, ${ }^{\mathrm{b}}$ University of Virginia Health System, Charlottesville, Va.

Michael D. Dake is a member of the W. L. Gore and Associates Endovascular Medical Advisory Board.

Received for publication May 5, 2008; revisions received July 23, 2008; accepted for publication Aug 2, 2008.

Address for reprints: Joshua D. Adams, MD, Department of Radiology, University of Virginia Health System, PO Box 800170, 1215 Lee St, Charlottesville, VA 22908

(E-mail: jda2d@virginia.edu).

J Thorac Cardiovasc Surg 2009;137:117-23

$0022-5223 / \$ 36.00$

Copyright $\subset 2009$ by The American Association for Thoracic Surgery

doi:10.1016/j.jtcvs.2008.08.004 formal oversight control and adherence to precisely defined inclusion and exclusion criteria for patient selection.

\section{PATIENTS AND METHODS}

\section{Study Design}

After approval by the Institutional Review Board for Health Sciences Research at the University of Virginia, a retrospective chart review of the first 50 consecutive patients who underwent thoracic aortic stent-graft therapy with the Gore TAG thoracic endoprosthesis (W. L. Gore \& Associates) after FDA approval was performed. This included all patients who underwent stent-graft placement from March 23, 2005, to September 8, 2006. Baseline demographics, coexisting medical conditions, and specific indications for endovascular repair were identified and recorded for each patient. Thoracic aortic aneurysms were characterized as an emergency if frank rupture or leak was imaged and urgent if the patient was symptomatic at the time of presentation. Thoracic aortic dissections were categorized as acute if repaired within 2 weeks of the initial onset of symptoms. Total length of aortic exclusion after stent-graft therapy was calculated from postoperative computed tomographic (CT) angiography using the centerline technique and recorded. Length of stay, number of intensive care unit (ICU) days, 30-day mortality, complications, and overall survival were specifically analyzed for each patient. The results of this analysis were then directly compared with the results of the phase II multicenter trial of the Gore TAG thoracic endoprosthesis ${ }^{2}$ to determine whether outcomes are significantly altered once the well-controlled inclusion and exclusion criteria of clinical trials are no longer strictly dictating patient selection.

\section{Procedures}

Patients were offered endovascular repair on the basis of suitability of aortic anatomy, specifically, proximal and distal landing zones of at least $20 \mathrm{~mm}$ in length with diameters greater than $20 \mathrm{~mm}$ and less than $40 \mathrm{~mm}$. Preoperative CT or magnetic resonance angiography was used to delineate the anatomy, guide proper device size selection, and identify 


\section{Abbreviations and Acronyms \\ $\mathrm{CSF}=$ cerebral spinal fluid \\ $\mathrm{CT}=$ computed tomography \\ $\mathrm{FDA}=$ Food and Drug Administration \\ $\mathrm{ICU}=$ intensive care unit}

TABLE 1. Patient characteristics

\begin{tabular}{lc}
\hline & No. of patients $(\%)$ \\
\hline Age (years) & $64.8 \pm 14.9$ \\
Male & $28(56)$ \\
Female & $22(44)$ \\
Comorbidities & \\
Hypertension & $35(70)$ \\
CRI & $6(12)$ \\
Hypercholesterolemia & $24(48)$ \\
CAD & $16(32)$ \\
COPD & $10(20)$ \\
CVA & $9(18)$ \\
PVD & $13(26)$ \\
Prior Aortic Surgery & $14(28)$ \\
Current Tobacco & $19(38)$ \\
\hline
\end{tabular}

$C R I$, Chronic renal insufficiency; $C O P D$, chronic obstructive pulmonary disease; $C A D$, coronary artery disease; $C V A$, cerebral vascular accident; $P V D$, peripheral vascular disease.

population included off-label indications such as ruptured aneurysm, type B aortic dissection, acute traumatic disruption, penetrating ulcer with or without intramural hematoma, thoracoabdominal aneurysm, aberrant right subclavian artery syndrome with dysphagia, exclusion of a left subclavian artery aneurysm, and repair of a large type I endoleak after stent-graft placement at an outside hospital. Thus, 19 $(38 \%)$ of the initial 50 patients treated after approval of the TAG device underwent stent-graft placement for a nonapproved indication.

\section{Procedure Characteristics}

Endovascular repair was successfully accomplished in all 50 patients. Procedure characteristics are detailed in Table 3. Twenty-one $(42 \%)$ patients required a conduit to either the common or external iliac artery or infrarenal abdominal aorta to bypass inadequate access vessels from the femoral region. In all others, the endografts were delivered through direct arteriotomy $(42 \%)$ or percutaneously $(16 \%)$ depending on the size and tortuosity of the femoral vessels and degree of atherosclerotic disease present. Only $15 \%(P<.05)$ of patients in the phase II trial had a conduit placed to facilitate introduction of the device. ${ }^{2}$

Zones of proximal aortic endograft attachment sites, as defined by Criado and associates, ${ }^{8}$ are depicted in Figure 1. Owing to inadequate length of the proximal landing zone, the left subclavian artery was covered in $52 \%$ of the study patients compared with $15 \%$ of patients in the phase II trial $(P<.05){ }^{2}$ Within our cohort, this included 20 patients with exclusion of the left subclavian artery only, 2 patients with exclusion of an aberrant right subclavian artery, 1 patient with exclusion of an aberrant left vertebral artery, 1 patient with planned exclusion of both the left subclavian and left common carotid arteries, and 2 patients with exclusion of all arch vessels with simultaneous arch reconstruction. In 1 patient with a type III thoracoabdominal aneurysm, the 
TABLE 2. Indications for thoracic aortic endovascular repair

\begin{tabular}{lcc}
\hline \multicolumn{1}{c}{ Indications } & \multicolumn{1}{c}{ No. of patients (\%) } \\
\hline Thoracic Aortic Aneurysms & $18(75)$ & \\
$\quad$ Elective & $3(12.5)$ & \\
$\quad$ Urgent & $3(12.5)$ & \\
$\quad$ Emergency & & $10(20)$ \\
Pseudoaneurysms & $6(60)$ & \\
$\quad$ Prior remote trauma & $4(40)$ & \\
$\quad$ Prior remote surgery & & $6(12)$ \\
Type B Dissections & $5(83)$ & \\
$\quad$ Acute & $1(17)$ & \\
$\quad$ Chronic & & $3(6)$ \\
Acute Traumatic Aortic Disruption & & $2(4)$ \\
Thoracoabdominal Aortic Aneurysms & & $2(4)$ \\
Penetrating Ulcers & & $1(2)$ \\
Aberrant Right Subclavian Artery Syndrome & & $1(2)$ \\
Left Subclavian Artery Aneurysm & & $1(2)$ \\
Type I Endoleak from Outside Hospital & & \\
\hline
\end{tabular}

celiac trunk and superior mesenteric artery were excluded after a debranching procedure. Preemptive left carotid-left subclavian bypass was performed in all 28 patients with exclusion of the left subclavian artery during the phase II trial; however, we used preemptive revascularization in only 6 (25\%) patients excluding the 2 who underwent planned arch vessel debranching procedures. Preemptive revascularization was performed for aberrant origin of the right subclavian artery in 2 patients, an incomplete posterior circulation in 2 patients, anomalous aortic origin of a dominant left vertebral artery in 1 patient, and stenosis of a codominant right vertebral artery in 1 patient. The patient with planned coverage of the left common carotid and subclavian arteries underwent a carotid-carotid bypass 1 day before stent-graft placement with no left subclavian revascularization.

At the discretion of the individual surgeon, spinal drains were placed preoperatively in $23(46 \%)$ patients based on factors that have been linked to increased rates of spinal ischemia: greater than $20 \mathrm{~cm}$ of total aortic exclusion, exclusion of the distal descending thoracic aorta, and history of prior aortic surgery. One patient had a spinal drain placed postoperatively for paraplegia.

TABLE 3. Procedure characteristics

\begin{tabular}{lc}
\hline & No. of patients $(\%)$ \\
\hline Aortic exclusion, mean $(\mathrm{cm})$ & $19.1 \pm 7.6$ \\
Left subclavian artery exclusion & $26(52)$ \\
Spinal drain & $23(46)$ \\
Delivery technique & \\
$\quad$ Percutaneous & $8(16)$ \\
Cutdown & $21(42)$ \\
$\quad$ Conduit & $21(42)$ \\
Concomitant procedures & $15(30)$ \\
Estimated Blood Loss, mean $(\mathrm{mL})$ & $201 \pm 249$ \\
\hline
\end{tabular}

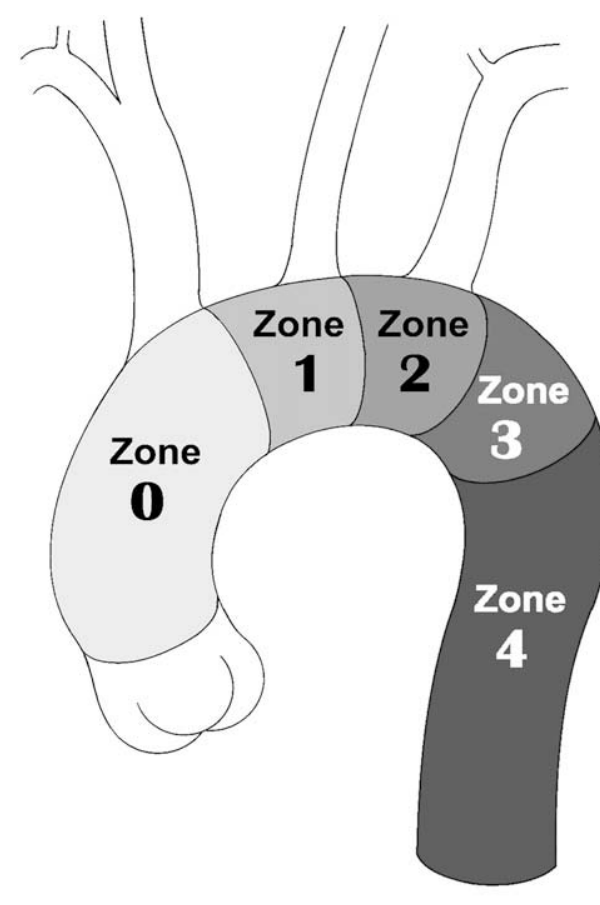

Zone $\mathbf{0}: \mathbf{n}=\mathbf{2}$

Zone 1: $\mathrm{n}=1$

Zone 2: $n=23$

Zone 3: $\mathrm{n}=12$

Zone 4: $n=12$

FIGURE 1. Aortic arch map depicting sites of proximal endograft deployment. $^{8}$

For the purpose of the study, concomitant procedures were defined as occurring during the same admission as the stent-graft procedure, not necessarily the same anesthesia event. Fifteen $(30 \%)$ patients underwent concomitant procedures, which ranged from endovascular procedures such as stenting of the renal or iliac arteries or embolization of the left subclavian artery to major surgical procedures such as aorta-bifemoral bypass or arch reconstruction.

\section{Outcomes}

Postoperative outcomes of our first 50 consecutive patients who underwent thoracic stent-graft placement with the TAG aortic endoprosthesis after FDA approval are compared with those of the phase II multicenter trial in Table 4. Specifically, there is no significant difference in number of ICU days, length of stay, or 30-day mortality. On average, study patients spent an extra day in the ICU compared with patients in the phase II trial; however, with the exclusion of the 3 patients who underwent stent-graft placement for acute traumatic disruption, all of whom had severe polytrauma requiring prolonged ICU and hospital care, the mean ICU stay decreased to $2.4 \pm 2.8$ days and the mean length of stay decreased to $5.3 \pm 4.7$ days. Both values are lower than those reported for the phase II trial. Within our patient group, there was 1 death within the first 30 postoperative days. This occurred in a 91-year-old woman who had a bradycardiac arrest while ambulating 7 days after successful endovascular 
TABLE 4. Outcomes: Post-FDA approval versus phase II trial $^{2}$

\begin{tabular}{lccc}
\hline & $\begin{array}{c}\text { Post-FDA, } \\
\mathbf{n}=\mathbf{5 0}(\%)\end{array}$ & $\begin{array}{c}\text { Phase II trial, } \\
\mathbf{n}=\mathbf{1 3 9}(\%)\end{array}$ & $\begin{array}{c}\boldsymbol{P} \\
\text { value }\end{array}$ \\
\hline Length of stay (d) & $7.5 \pm 10.9$ & $7.6 \pm 18$ & .97 \\
ICU stay (d) & $3.7 \pm 7.3$ & $2.6 \pm 14.6$ & .61 \\
30-Day mortality & $1(2)$ & $2(1.5)$ & .68 \\
\hline
\end{tabular}

$F D A$, Food and Drug Administration; $I C U$, intensive care unit.

exclusion of a leaking thoracic aortic aneurysm. At 1 year, overall survival in study patients was $92 \%$ compared with $82 \%$ in the phase II trial $(P=.16){ }^{2}$

A comparison of early complications, defined as those occurring in the first 30 days after the procedure, is detailed in Table 5. With the exception of a higher endoleak rate in our post-FDA approval group, no statistically significant differences were identified. The complications included small type I endoleaks visualized on postdeployment aortography while the patient was fully heparinized. Isolated type I endoleaks were identified in 7 patients. Four resolved spontaneously by 6 months, 1 persisted with no sac enlargement and has been observed, 1 was associated with sac enlargement on CT angiography and was converted to open surgical repair, and 1 was related to proximal endograft collapse that was successfully treated endovascularly with placement of a balloon expandable stent. Type II endoleaks were identified in 3 patients with no evidence of sac enlargement and have therefore been observed. Finally, 2 patients demonstrated both type I and II endoleaks. In both cases the type I endoleak resolved spontaneously. One patient's type II endoleak has been observed inasmuch as there has been no sac enlargement, and 1 patient underwent successful transcatheter endoleak embolization with Onyx liquid embolic agent (ev3, Irvine, Calif) when slight sac enlargement was detected on CT angiography.

Spinal cord injury resulting in permanent paraplegia occurred in $1(2 \%)$ patient who had a ruptured thoracic aortic aneurysm with persistent hypotension despite vasopressor therapy preoperatively. Owing to the emergency nature of

TABLE 5. Early complications: Post-FDA approval versus phase II trial $^{2}$

\begin{tabular}{lccr}
\hline Complications $<\mathbf{3 0}$ days & $\begin{array}{c}\text { Post-FDA, } \\
\mathbf{n}=\mathbf{5 0}(\%)\end{array}$ & $\begin{array}{c}\text { Phase II trial, } \\
\mathbf{n}=\mathbf{1 3 9}(\%)\end{array}$ & $\begin{array}{c}\boldsymbol{P} \\
\text { value }\end{array}$ \\
\hline Any major & $17(34)$ & $45(32)$ & .93 \\
Bleeding & $5(10)$ & $12(9)$ & .94 \\
Endoleak & $12(24)$ & $5(4)$ & $<.01$ \\
Vascular & $5(10)$ & $20(14)$ & .63 \\
Spinal cord injury & $1(2)$ & $4(3)$ & .89 \\
CVA/TIA & $2(4)$ & $5(4)$ & .67 \\
Pulmonary & $2(4)$ & $14(10)$ & .31 \\
Cardiac & $4(8)$ & $4(3)$ & .28 \\
Death & $1(2)$ & $2(1.5)$ & .68 \\
\hline FDA, Food and Drug Administration; $C V A$, cerebrovascular accident; TIA, transient \\
ischemic attack.
\end{tabular}

repair, no spinal drain was placed preoperatively and the patient's aneurysm required greater than $20 \mathrm{~cm}$ of aortic exclusion. Other neurologic complications included a cerebrovascular accident in $2(4 \%)$ patients. In both cases the cause was believed to be embolic. Both patients had significant manipulation of their aortic arch with exclusion of at least one arch vessel. Both patients' symptoms resolved during their admission.

Although not statistically significant, there were fewer vascular complications in the post-FDA approval group $(8 \%)$ than in the phase II trial group $(20 \%)$. Two vascular complications related to access occurred, including one failed percutaneous closure device, which required arterial exposure and primary surgical closure, and an injury to the right common iliac artery detected during removal of the delivery catheter, which was treated with an endovascular stent. Additionally, 2 patients treated for dissection were found to have persistent patency and retrograde filling of the thoracic false lumen with aortic enlargement. In 1 patient's case, the false lumen extended into the left renal and left common iliac arteries. This was successfully treated endovascularly with placement of stents bridging the true lumen of the aorta and the respective arteries with obliteration of the false lumen within the branches.

Figure 2 demonstrates a Kaplan-Meier survival curve showing freedom from overall and stent-graft-related mortality. With a mean follow-up of 625.1 days, overall survival was $88 \%$ and freedom from stent-graft-related death was $100 \%$. Of the 6 nonsurvivors, 3 died of cancer-related causes, 2 of cardiac-related causes, and 1 patient of pneumonia.

Table 6 compares late complications, those occurring greater than 30 days after the procedure, between the two groups and demonstrates no statistically significant

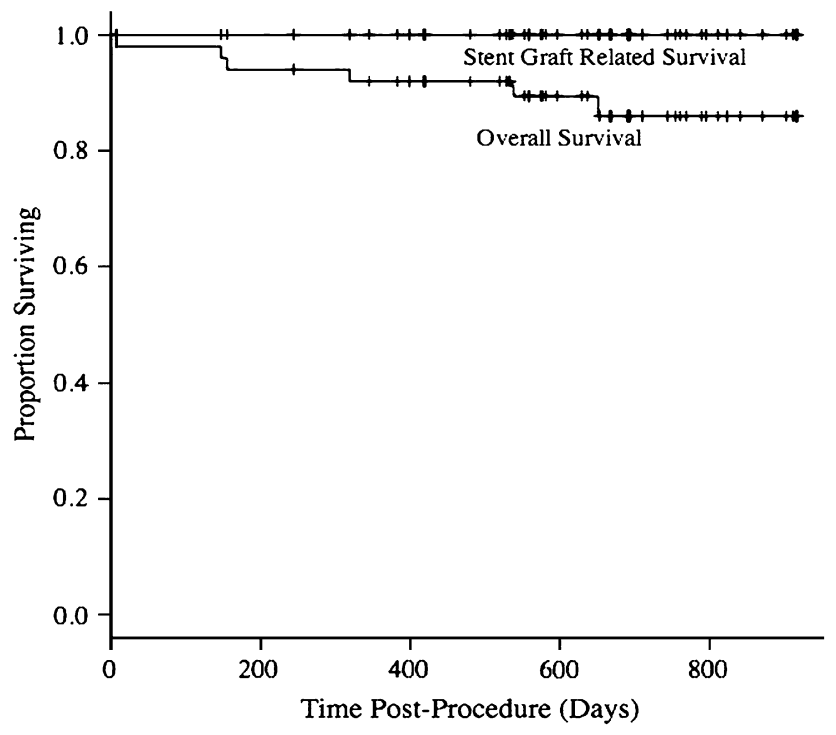

FIGURE 2. Kaplan-Meier curves of stent-graft-related and overall survival. 
TABLE 6. Late complications (1-year follow-up): Post-FDA approval versus phase II trial ${ }^{2,16}$

\begin{tabular}{lccc}
\hline \multicolumn{1}{c}{ Late events } & $\begin{array}{c}\text { Post-FDA, } \\
\mathbf{n}=\mathbf{5 0}(\%)\end{array}$ & $\begin{array}{c}\text { Phase II trial, } \\
\mathbf{n}(\%)\end{array}$ & $\begin{array}{c}\boldsymbol{P} \\
\text { value }\end{array}$ \\
\hline Migration, proximal & 0 & $0 / 97(0)$ & .999 \\
Migration, distal & 0 & $1 / 84(1)$ & .67 \\
Endoleak rate & $8(16)$ & $7 / 97(7)$ & .15 \\
Conversion to open & $1(2)$ & $1 / 109(1)$ & .81 \\
Endovascular revision & $3(6)$ & $1 / 109(1)$ & .19 \\
1-Year overall survival & $46(92)$ & $89(82)$ & .16 \\
\hline
\end{tabular}

FDA, Food and Drug Administration.

difference regarding migration, endoleak rate, or need for further intervention at 1 year. In the post-FDA group, only one new endoleak developed during the follow-up interval. This was either a type II or III endoleak with no change in aortic diameter and has therefore only been observed.

\section{DISCUSSION}

The history of endovascular repair of descending thoracic aneurysms has been well documented since first reported in a high-risk population by Dake and associates ${ }^{1}$ in 1994. Although complication rates were relatively high with this first generation of devices, the development of multiple commercially manufactured endografts with improved flexibility and durability markedly decreased the number of complications. ${ }^{2-5}$ Many groups have since reported superior shortterm and midterm results to those of conventional open surgery, including decreased operative time, shorter ICU and hospital stays, and lower perioperative morbidity and mortality rates., 90

In March of 2005, after an advisory panel review of the results of the phase II multicenter trial with the TAG endoprosthesis, ${ }^{2}$ the FDA approved the device to become the first commercially available endograft in the United States to treat descending thoracic aneurysms. After this decision, clearly mandated training instructions for use accompanied the commercialization process; however, the well-controlled inclusion and exclusion criteria that governed patient selection in the clinical trials could no longer be strictly enforced. Off-label indications have rapidly expanded to include additional thoracic aortic diseases such as ruptured aneurysm, type B aortic dissection, traumatic aortic disruption, complicated penetrating ulcer, intramural hematoma, and treatment of lesions related to connective tissue disorders. The current report describes our single-center, "real world" experience with this device since it received FDA approval and favorably compares our results with those of the phase II multicenter trial.

Similar comparisons have been made in the literature regarding endovascular repair of abdominal aortic aneurysms, including two separate studies that have compared their post-FDA approval single-center experiences with that of the overall multicenter trials data. ${ }^{11,12}$ Both studies reported no significant differences in complication and mortality rates between the trial groups and the post-FDA approval groups; however, they concluded that extending endovascular indications to treat increasingly complex aortic aneurysms, including those with short, angulated necks, had resulted in increased numbers of type I endoleaks. Further, Adelman and colleagues ${ }^{12}$ demonstrated that significantly more patients in the post-FDA approval group required additional access procedures such as iliac angioplasty or stenting, conduits, or either iliofemoral or femorofemoral bypass to introduce the device. Although we report similar findings in our own post-FDA experience with the TAG device, endovascular treatment of the thoracic aorta differs from its abdominal counterpart in that thoracic endovascular therapy may be applied to multiple aortic diseases, not just aneurysms.

Although the phase II trial investigated thoracic aortic stent grafts for the treatment of descending thoracic aneurysms only, the indications for treatment in the post-FDA approval study group included multiple aortic diseases. The distribution of individual aortic pathologic conditions is similar to that of Verhoye and coworkers, ${ }^{6}$ who reported a nearly 5-year experience with thoracic stent grafting at two centers in France. The authors sought to evaluate outcomes based on acute versus chronic disease entities. In comparison with our study, similar perioperative morbidity and mortality were reported using three different stent-graft systems (Talent, Medtronic, Inc, Santa Rosa, Calif; TX2, Cook, Inc, Bloomington, Ind; and the TAG, W. L. Gore \& Associates, Inc, Flagstaff, Ariz). A higher endoleak rate $(29.6 \%$ vs $16 \% ; P=.16)$ was reported in their study, the majority occurring in the chronic pathology group. They concluded that in their hands, thoracic aortic stent-graft therapy was particularly effective in the treatment of acute diseases such as complicated acute type B dissections, acute traumatic rupture, and symptomatic penetrating ulcers, as well as chronic pseudoaneurysms from prior surgery or trauma.

Less common indications for endograft placement in the thoracic aorta may include purposeful exclusion of vascular abnormalities involving various aortic branches. In our series, an endograft was successfully used to exclude a retroesophageal aberrant right subclavian artery causing severe dysphagia, as well as a proximal left subclavian artery aneurysm in another patient.

When comparing our early adverse events to those of the phase II trial, we observed a significantly higher endoleak rate $(24 \%$ vs $4 \% ; P<.01)$. During intraoperative aortography or in the early postoperative period, we noted more proximal type I endoleaks (8) than reported in the phase II trial (1), with nearly equal type II endoleaks. It is likely that this difference is due to our willingness to challenge more hostile aortic arches not permitted by anatomic constraints dictated by clinical trial protocols. This is supported by the fact that $6 \%$ of patients had the proximal endograft attachment site in zones 0 and 1 (which was not 
allowed in the phase II trial) and that we were more often required to exclude the left subclavian artery $(52 \%$ vs $15 \%$ in the phase II trial; $P<.0001)$ to obtain a dimensionally suitable proximal landing zone. As previously discussed, this tends to place the proximal aspect of the endograft into the horizontal segment of the aortic arch, thereby increasing the chances for poor opposition of the endograft along the inner curve of the aortic arch apex, which may promote creation of a perigraft endoleak channel. Ideas for future devices to combat this problem may include flared endografts or smaller articulations that would allow the endograft to conform better to the natural arch angle.

An additional topic related to coverage of the left subclavian artery that warrants further discussion is our approach to revascularization after exclusion. As previously reported, we used a policy of selective revascularization in contrast to the phase II trial, in which a bypass was performed universally in conjunction with planned exclusion of the left subclavian artery. Excluding planned debranching procedures, this series included adjunctive left subclavian artery bypass or transposition in $6(25 \%)$ patients. After intentional left subclavian occlusion without associated surgical revascularization, 3 patients had left arm effort discomfort. Late revascularization was required in 2 of the 3 additional patients $(11 \%)$. One patient's symptoms resolved after cardiac rehabilitation. Interestingly, the 2 patients requiring bypass were both women, under 40 years of age, and treated for a pseudoaneurysm.

Neurologic complications continue to be a rare but devastating complication of thoracic aortic stent grafting. Spinal cord ischemia, both immediate and delayed, resulting in paraplegia has been reported in multiple series. In these, its occurrence most commonly ranges from $3 \%$ to $5 \%$ and appears to be a multifactorial event. ${ }^{13}$ As previously stated, our single episode of spinal cord injury developed in the immediate postoperative period after emergency repair of a ruptured thoracic aortic aneurysm and did not respond to delayed CSF drainage, steroid administration, and aggressive blood pressure control. Multiple risk factors have been implicated, including an aortic treatment length greater than $20 \mathrm{~cm}$, coverage of the distal thoracic aorta between the T8 and L2 vertebral levels, and a history of prior abdominal aortic repair. ${ }^{14}$ Perioperative hypotension, defined as a mean arterial pressure less than $70 \mathrm{~mm} \mathrm{Hg}$, has also been implicated as a risk factor for both immediate and delayed spinal cord injury. ${ }^{15}$ Like many authors, we advocate the use of prophylactic CSF drainage in patients with these preoperative risk factors and closely manage blood pressure in the perioperative period to maintain a mean arterial pressure greater than $90 \mathrm{~mm} \mathrm{Hg}$.

Strokes have also been reported after thoracic stent-graft procedures, especially in patients requiring extensive aortic arch manipulation. ${ }^{2}$ Identical to the frequency detailed for the phase II trial, we report a $4 \%$ rate of stroke. Within both groups, strokes occurred almost exclusively in patients requiring coverage of the left subclavian artery. This is likely not the only factor inasmuch as the stroke rates were identical despite the notable increased frequency of arch vessel coverage in our group (52\%) compared with that in the phase II report $(15 \%)$. Risks of embolic cerebrovascular events are likely related to the severity and composition of the aortic atherosclerotic plaques and the extent of wire, catheter, and device manipulation within the arch.

Finally, vascular access to the thoracic aorta continues to be a significant issue with thoracic aortic endografting. Vascular complications related to access were more frequently reported in the phase II trial than in our post-FDA approval group ( $14 \%$ vs $10 \% ; P=.63)$, although this did not reach statistical significance. Contributing to this, as the phase II authors pointed out, is an appreciation of the critical value of using a graft conduit as a preemptive measure rather than a bailout after a vascular injury has occurred. ${ }^{2}$ In our post-FDA approval group, a conduit for arterial access was used in $42 \%$ of patients ( 5 men, 16 women), emphasizing the more frequent need in women secondary to smaller iliac and femoral vessels.

\section{CONCLUSION}

The outcomes of this study demonstrate that in the postFDA approval era of the TAG endoprosthesis, we are achieving similar results to those of the phase II multicenter trial as we apply endovascular repair to a wider range of thoracic aortic diseases and more difficult aortic and iliac anatomy. Although this study has limitations, including those of being a nonrandomized, retrospective review that is subject to inherent bias, we believe the data further contribute to the ever-growing body of evidence supporting the use of thoracic stent grafts for a variety of thoracic aortic lesions.

We thank Diane Washington for her assistance in preparing this manuscript and Donald L. Persson for providing the illustration presented in Figure 1.

\section{References}

1. Dake MD, Miller DC, Semba CP, Mitchell RS, Walker PJ, Liddell RP. Transluminal placement of endovascular stent-grafts for the treatment of descending thoracic aortic aneurysms. N Engl J Med. 1994;331:1729-34.

2. Makaroun MS, Dillavou ED, Kee ST, Sicard G, Chaikof E, Bavaria J, et al. Endovascular treatment of thoracic aortic aneurysms: results of the phase II multicenter trial of the GORE TAG thoracic endoprosthesis. J Vasc Surg. 2005;41:1-9.

3. Greenberg R, Resch T, Nyman U, Lindh M, Brunkwall J, Brunkwall P, et al. Endovascular repair of descending thoracic aortic aneurysms: an early experience with intermediate-term follow-up. J Vasc Surg. 2000;31:147-56.

4. Czerny M, Grimm M, Zimpfer D, Rodler S, Gottardi R, Hutschala D, et al. Results after endovascular stent-graft placement in atherosclerotic aneurysms involving the descending aorta. Ann Thorac Surg. 2007;83:450-5.

5. Dagenais F, Shetty R, Normand JP, Turcotte R, Mathieu P, Voisine P. Extended applications of thoracic aortic stent-grafts. Ann Thorac Surg. 2006; 82:567-72.

6. Verhoye JP, de Latour B, Heautot JF, Vola M, Langanay T, Corbineau H, et al. Mid-term results of endovascular treatment for descending thoracic aorta diseases in high-surgical risk patients. Ann Vasc Surg. 2006;20:714-22. 
7. Ince H, Rehders TC, Petzsch M, Kische S, Nienaber CA. Stent-grafts in patients with Marfan syndrome. J Endovasc Ther. 2005;12:82-8.

8. Criado F, Abul-Khoudoud O, Domer G, McKendrick C, Zuzga M, Clark N, et al. Endovascular repair of the thoracic aorta: lessons learned. Ann Thorac Surg. 2005;80:857-63.

9. Ehrlich M, Grabenwoeger M, Cartes-Zumelzu F, Grimm M, Petzel D, Lammer J, et al. Endovascular stent-graft repair for aneurysms of the descending thoracic aorta. Ann Thorac Surg. 1998;66:19-25.

10. Mitchell R, Dake M, Semba C, Fogarty T, Zarins C, Liddell R, et al. Enodvascular stent-graft repair of thoracic aortic aneurysms. J Thorac Cardiovasc Surg. 1996; 111:1054-62

11. Ramaiah V, Westervand A, Thompson C, Ravi R, Rodriguez J, DiMugno L, et al. The AneuRx stent-graft since FDA approval: single-center experience of 230 cases. J Endovasc Ther. 2002;9:464-9.
12. Adelman M, Rockman C, Laparello P, Jacobowitz G, Tuerff S, Gagne P, et al Endovascular abdominal aortic aneurysm (AAA) repair since the FDA approval: are we going too far? J Cardiovasc Surg. 2002;43:359-67.

13. Gravereaux EC, Faries PL, Burks JA, Latessa V, Spielvogel D, Hollier LH, et al Risk of spinal cord ischemia after endograft repair of thoracic aortic aneurysms. J Vasc Surg. 2001;34:997-1003.

14. Baril DT, Carroccio A, Ellozy SH, Palchic E, Addis MD, Jacobs TS, et al. Endovascular thoracic aortic repair and previous or concomitant abdominal aortic repair: is the increased risk of spinal cord ischemia real? Ann Vasc Surg. 2006;20:188-94.

15. Chiesa R, Melissano G, Marrocco-Trischitta MM, Civilini E, Setacci F. Spinal cord ischemia after elective stent-graft repair of the thoracic aorta. $J$ Vasc Surg. 2005;42:11-7.

16. GORE TAG thoracic endoprosthesis annual clinical update. Flagstaff (AZ): W.L. Gore \& Associates, Inc; 2007 Apr. 